\title{
The Online Follow-Up Framework for Neutrino Triggered Alerts from IceCube
}

\author{
The IceCube Collaboration ${ }^{1}$ \\ ${ }^{\dagger}$ http://icecube.wisc.edu/collaboration/authors/icrc15_icecube \\ E-mail: alexander.stasikedesy.de
}

\begin{abstract}
The current operation of online programs for sending follow-up alerts to optical, X-ray and gamma-ray telescopes shows the feasibility of neutrino-triggered multi-messenger astronomy. Building on the experience of these programs, we generalize the approach and merge them into a combined generic framework. The upgrade consists of a single event stream selected at the South Pole and transmitted north, where an online search for transients and flaring points is performed. The event selection will be compared to established offline point source searches in IceCube. Performance of the stream in terms of delay and stability will be shown.
\end{abstract}

Corresponding authors: Alexander Johannes Stasik ${ }^{1 *}$, Thomas Kintscher ${ }^{1}$, Marek Kowalski ${ }^{1,2}$, Elisa Bernadini ${ }^{1,2}$

${ }^{1}$ Deutsches Elektronen-Synchrotron (DESY), Platanenallee 6, D-15735 Zeuthen, Germany

${ }^{2}$ Institut für Physik, Humboldt Universität, Newtonstr. 15, D-12489 Berlin, Germany

The 34th International Cosmic Ray Conference,

30 July- 6 August, 2015

The Hague, The Netherlands

${ }^{*}$ Speaker. 


\section{Introduction}

Neutrinos play an important role in modern astrophysics due to their complementary nature to other messengers like photons and cosmic rays [1]. Neutrinos only interact through the weak force making them a natural choice for astronomy since they are neither deflected by magnetic fields nor absorbed between source and observer and thus point back to their origin. The production of high energetic neutrinos is associated with hadronic interactions and thus is directly linked to cosmic rays. This makes neutrinos a viable tool to search for sources of the highest energy cosmic rays. However, detection of astrophysical neutrinos is very challenging due to their small cross section and the large background of atmospheric muons and atmospheric neutrinos.

While the angular resolution of neutrino telescopes is orders of magnitude worse than modern optical telescopes, they have a field of view that can cover the entire sky with a duty cycle of almost $100 \%$. This complementary nature of neutrino telescopes and typical electromagnetic telescopes motivates the combination of these channels. IceCube has shown the existence of astrophysical neutrinos, but no sources have yet been identified $[2,3]$.

While for steady sources combining different messenger channels like neutrino and electromagnetic data is possible with archival data, transient sources benefit strongly from real time follow-up observation with small field-of-view optical telescopes. Thus external real time triggers are required. IceCube with its all-sky field-of-view can provide such triggers. The typical time scales of potential sources vary between several hundred days for supernovae or flaring AGNs [4] and go down to several seconds for short GRBs [5]. These Neutrino triggered Target of Opportunity $(\mathrm{NToO})$ programs require highly automated and stable systems since the entire analysis, from identifying an interesting event to notifying follow-up observatories and changing observation schedules, has to be done in seconds and possibly even without a human being involved in the loop.

IceCube plays a central role as a triggering facility in many multi-messenger efforts. Different follow-up programs like optical (OFU) [6], X-ray follow-up (XFU) [7] and gamma follow-up (GFU) [8] have been independently running for several years [9]. Under these programs, alerts have been send to PTF [10], the Swift satellite [11], MAGIC [12], Veritas [13] and the multimessenger network AMON [14]. These systems analyze the neutrino data in real time at the South Pole. Only follow-up alerts leave the Pole in real-time while the sub-threshold data stays at the detector and is only available for offline studies. This paper describes the design and capabilities of the new IceCube real time system. The new system provides for the first time a stream of high quality events in real time available in the north with a latency of a few tens of seconds. This sub-threshold stream offers many new opportunities in multi-messenger astronomy [14]. Section 2 will describe the IceCube neutrino telescope. Section 3 will present the design behind the real time system running in IceCube. Section 4 will show the sensitivity for the event selections available in near real time in the current system.

\section{The IceCube Neutrino Telescope}

IceCube is a cubic-kilometer neutrino detector installed in the ice at the geographic South Pole between depths of $1450 \mathrm{~m}$ and $2450 \mathrm{~m}$. Detector construction started in 2005 and finished in 
2010. Neutrino reconstruction relies on the optical detection of Cherenkov radiation emitted by secondary particles produced in neutrino interactions in the surrounding ice or the nearby bedrock [15]. Neutrinos can interact and produce secondary particles via charged current (CC) or neutral current (NC) interactions. While $\mathrm{NC}$ interactions produce a localized hadronic cascade inside the ice, $\mathrm{CC}$ interactions produce an additional lepton corresponding to the initial neutrino flavor. Electrons and tau leptons typically lose their energy on length scales of several meters, normally indistinguishable from the hadronic cascade. $\mathrm{NC}$ and $\mathrm{CC}$ neutrino interactions caused by electron or tau neutrinos produce localized, almost spherical signatures inside the detector. These event signatures are called cascades. While their directional reconstruction is challenging due to their almost spherical shape, energy estimation is robust as long as the cascade is contained inside the detector, enabling a calorimetric measurement. Muons produced in $v_{\mu} \mathrm{CC}$ interactions, on the other hand, can travel many kilometers in ice and produce light along their way. These events are called tracks and provide good angular reconstructions at about one degree [16]. The track length can exceed the detector boundary, thus energy reconstructions are challenging since only the deposited energy inside the detector can be measured. At the neutrino energy of interest, the small kinematic angle between muon neutrino and muon is below the detector resolution and can be neglected [17]. Since track events are better suited for neutrino astronomy than cascades due to their better angular resolution, the following discussion is limited to $v_{\mu} \mathrm{CC}$ events.

Event selection starts from the online muon filter selection that identifies high quality tracks with a rate of about $40 \mathrm{~Hz}$. This rate is dominated by atmospheric muons. To increase the neutrino purity of the sample, more advanced and more time consuming reconstructions are required. Since computing power is limited at the South Pole, these reconstructions can only be applied to a subset of the events. The Online Level 2 Filter uses the outcome of a likelihood reconstruction to further reduce the contamination from atmospheric muons. This likelihood reconstruction takes into account the actual photon propagation to the optical modules in the detector. Selection criteria are then, e.g., quality of the likelihood fit and the total number of modules detecting photons. After application of these criteria, the event rate is reduced to $5 \mathrm{~Hz}$. More time-consuming reconstructions are applied afterwards, using a more advanced model for light propagation in the ice. They also explore the likelihood space to give an error on the angular reconstruction. Based on these reconstructions, a multivariate classifier is used for the final filter. At the end, the event rate is several $\mathrm{mHz}$ and has a neutrino purity of about $90 \%$.

\section{Online System}

The goal of the IceCube real time system is to provide a system that can generate followup notifications called alerts with a minimal delay after one or more neutrinos are detected. The rate of false alerts should be as low as possible without losing signal events in the process. Also required are high stability and reliability since the entire system must run autonomously. Based on the event selection described in Section 2 a system has been built to transmit these events to the north, analyze them in real time and generate follow-up alerts to be send to other telescopes. The events are also used by IceCube for, e.g., real time event displays for detector monitoring purposes. The online system can handle any kind of event stream that is generated in real time at the Pole, 


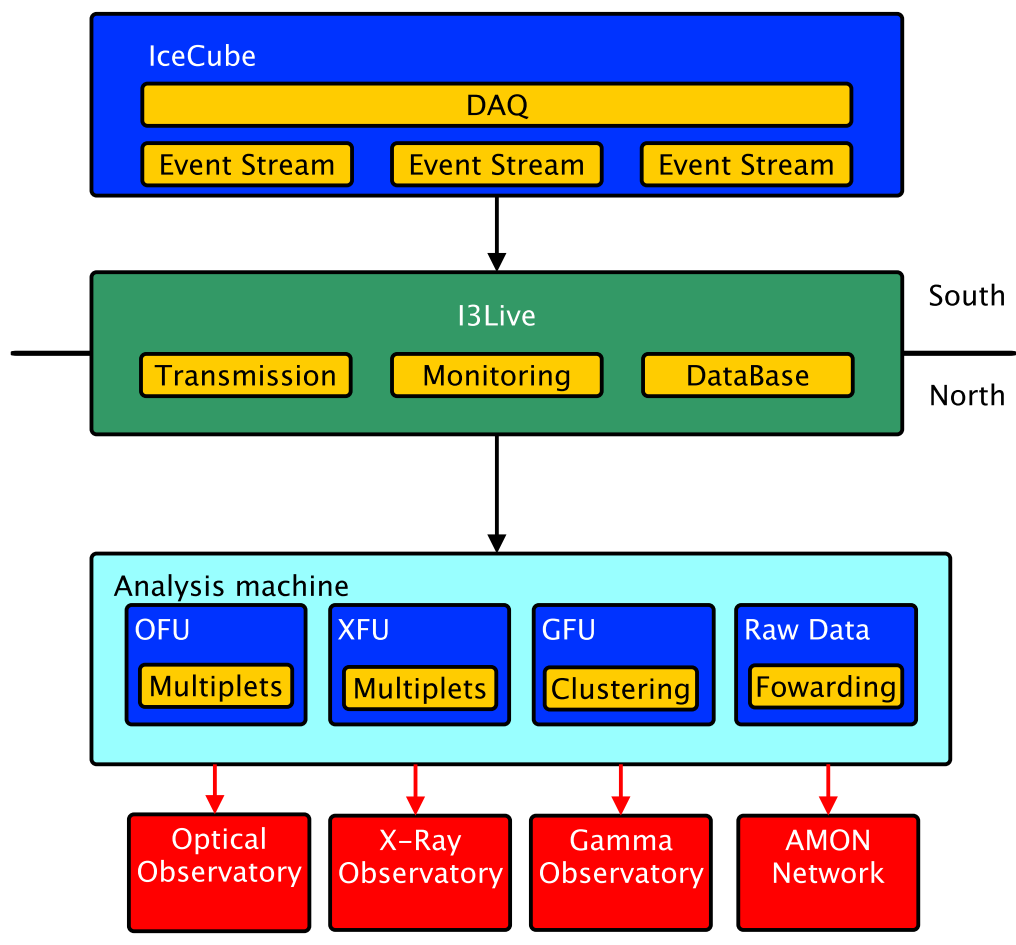

Figure 1: Sketch of the IceCube real time system. Black arrows indicate flow of event information and red arrows flow of alert information.

but since tracks from $v_{\mu} \mathrm{CC}$ events are the starting point of current online systems, discussion will focus here on this stream.

Once an event passes the filtering process described in Section 2, the full event information is stored on disk at the Pole. For data transmission to the north, IceCube uses several high bandwidth satellites which can transfer up to $100 \mathrm{~GB}$ per day. Due to the remote location of IceCube, these satellites are only available a few hours per day and thus are not suited for real time operations. To control and monitor the detector in real time, IceCube uses the I3Live system. I3Live has an instance running at South Pole and a mirrored version in the north. Both communicate using the Iridium satellite network [18]. Iridium satellites have a low bandwidth, but are available 24/7 with a latency of several seconds. The I3Live system at the South Pole is constantly listening to the stream of events exiting the filtering and processing system. If an event is marked as to have passed the real time event selection, it is picked up by I3Live and transmitted to the north. Due to bandwidth limitations it is not possible to transmit the entire event information, so just event direction, error estimator, energy estimator, event time and reconstruction quality parameters are sent north.

As the events arrive in the north, they are stored in a data base and forwarded to a dedicated machine that hosts the real time system in the north. This system consists of a set of follow-up clients each of which is dedicated to a specific set of events, a certain analysis method and a special follow-up facility to which it sends alerts. A software package is provided that includes generic functionality like interacting with the I3Live database, stability and monitoring tools for the client and tools like coordinate transformations and filter functions. Since every follow-up client will need 
this functionality, the toolbox provided makes it easy to write a new client without knowledge of the technical side of the system. Figure 1 shows a sketch of the full python-based system. The system is modular so that additional channels (like e.g. the high energy starting event selection HESE [19] in real time) can just be added if they meet the computational and bandwidth requirements. It is also possible to simply add additional follow-up clients.

The transmission of single events has been since the beginning of this year. The delay is of the order of several seconds with some single outliers due to satellite connection problems. Figure 3 shows that the median delay is about $22 \mathrm{~s}$ and after $30 \mathrm{~s}$ about $99 \%$ of the events arrived.

\section{Event selection}

The main channel for triggering follow-up operations are $v_{\mu} \mathrm{CC}$ muon tracks due to their superior angular resolution. As described in Section 2, the muon track event selection has a final rate of about $5 \mathrm{mHz}$. Figure 2 shows the time-independent point source sensitivity of one year data processed as in the real time event stream to a data-set processed with offline algorithms. The offline data-set shows slightly better performance than the real time stream. This is a result of better, but much more time consuming, event reconstructions. The real time data, which is nearly as good as the offline data, can be processed in real time, decreasing latency from days to seconds.

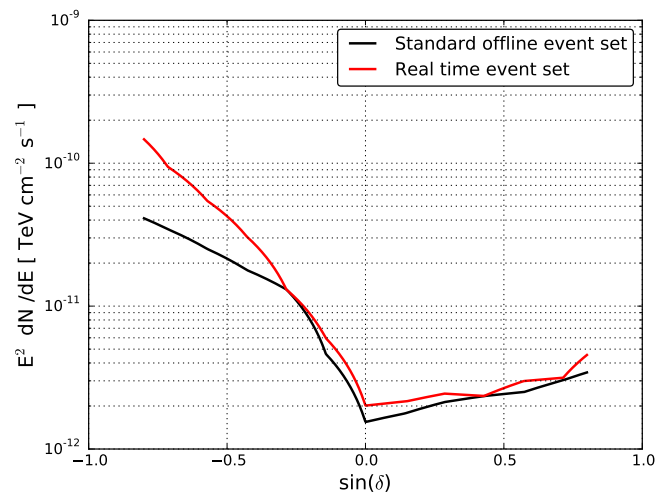

Figure 2: Time independent point source sensitivities for the online data stream compared to an offline event selection, 322 days livetime [20].

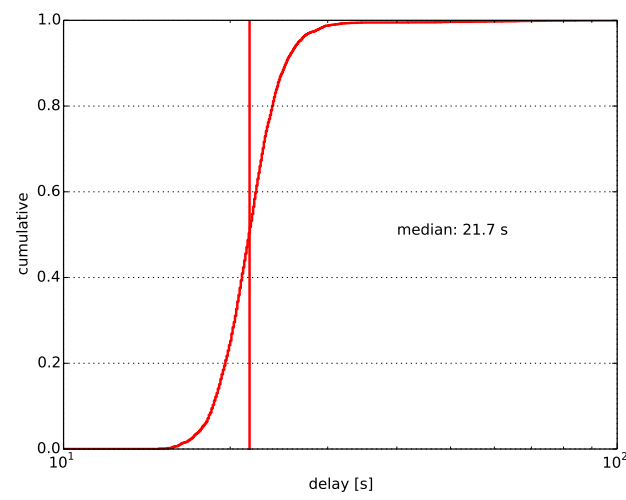

Figure 3: Cumulative distribution of delays between triggering time of the event and arrival time in the north.

\section{Summary and outlook}

The IceCube real time system provides a framework to process, transmit and analyze neutrino events with a typical latency of 20 to 30 seconds. The system is modular so that additional followup clients or event selections can be added if they can be filtered and reconstructed in real time at the Pole. The current implementation included high quality track like event stream. The point source sensitivity is slightly below the comparable offline data-set as expected due to computational limitations in real time. 


\section{References}

[1] T. K. Gaisser and T. Stanev, Astroparticle Physics 39 (Dec., 2012) 120-128, [arXiv: 1202 . 0310 ].

[2] IceCube Collaboration, M. Aartsen et al., Science 342 (2013) 1242856, [arXiv: 1311 . 5238].

[3] M. Kowalski, ArXiv e-prints (Nov., 2014) [arXiv: 1411.4385 ].

[4] A. M. Atoyan and C. Dermer, New Astron.Rev. 48 (2004) 381-386, [ast ro-ph/ 0402646 ].

[5] E. Waxman and J. Bahcall, Phys. Rev. Lett. 78 (Mar, 1997) 2292-2295.

[6] IceCube, ROTSE Collaboration, R. Abbasi et al., Astron.Astrophys. 539 (2012) A60, [arXiv:1111.7030].

[7] P. Evans, J. Osborne, J. Kennea, M. Smith, D. Palmer, et al., Mon.Not.Roy.Astron.Soc. 448 (2015), no. 3 2210-2223, [arXiv:1501.04435].

[8] IceCube Collaboration, M. Aartsen et al., arXiv:1309.6979.

[9] IceCube Collaboration, PoS(ICRC2015)1052, these proceedings (2015).

[10] N. M. Law et al., PASP 121 (Dec., 2009) 1395-1408, [arXiv:0906.5350].

[11] N. Gehrels et al., Astrophysical Journal 611 (Aug., 2004) 1005-1020.

[12] C. Baixeras, Nuclear Physics B - Proceedings Supplements 114 (2003), no. 0247 - 252. Proceedings of the XXXth International Meeting of Fundamentals Physics.

[13] T. C. Weekes et al., Astroparticle Physics 17 (May, 2002) 221-243, [astro-ph / 0108478 ].

[14] AMON Collaboration, M. W. E. Smith et al., Astroparticle Physics 45 (May, 2013) 56-70, [arXiv:1211.5602].

[15] IceCube Collaboration, A. Achterberg et al., Astropart.Phys. 26 (2006) 155-173, [astro-ph/0604450].

[16] IceCube Collaboration, M. G. Aartsen et al., Nuclear Instruments and Methods in Physics Research A 736 (Feb., 2014) 143-149, [arXiv:1308.5501].

[17] ANTARES Collaboration, , ArXiv Astrophysics e-prints (July, 1999) [astro-ph/9907432].

[18] Iridium communications inc., www.iridium.com.

[19] IceCube Collaboration, IceCube Collaboration, Science 342 (Nov., 2013) 1, [arXiv:1311. 5238 ].

[20] IceCube Collaboration, M. Aartsen et al., arXiv:1406.6757. 